\title{
Biofilms, Minerals, and Bronchioles: Understanding Microenvironments Through Correlative Microscopy.
}

\author{
R.C. Hunter $* * *$ \\ * Department of Biology, Massachusetts Institute of Technology, Cambridge, MA 02139 \\ ** Molecular and Cellular Biology, University of Guelph, Guelph, Ontario N1G 2W1
}

Only recently have we recognized bacteria as major players in global geochemistry. Similarly, the recognition of biofilms as the predominant mode of microbial growth in nature has only been a recent finding. Because the two fields are in their infancy, we understand very little about the interplay between biofilms and the geosphere. In particular, there is a need for understanding the spatial organization of microenvironments within biofilms that contribute to the complexity of metal-microbe interactions. We have focused on a correlative microscopy approach to investigate these processes. For example, confocal microscopy was used together with several fluorophores to reveal that mature Pseudomonas biofilms are oxygen-saturated throughout, but feature remarkable $\mathrm{pH}$ changes ( $\sim 2$ units) over micron-sized areas [Fig. 1a] [1]. In addition, cryo-electron microscopy has revealed that the spatial organization of cells and exopolymers is extraordinarily complex at both cellular and extracellular levels [Fig 1b] [2]. To further understand the influence of this heterogeneity on mineral development, geochemical imaging is also required. Here, we combine the advantages of cryo-EM with scanning transmission $\mathrm{x}$-ray microscopy (STXM) to investigate the speciation and partitioning of iron throughout the same biofilm community [3].

A novel cryo-fixation/freeze-drying technique was employed to prepare STXM biofilm samples. Importantly, we show that this method avoids known limitations of polymer extraction and re-distribution throughout sample processing (Fig. 2a). Importantly, when these samples were imaged using C1s edge STXM sequences, we confirmed a heterogeneous distribution of biological material throughout $P$. aeruginosa biofilms. Additionally, quantitative mapping using $\mathrm{Fe}$ adsorption edges revealed that both $\mathrm{Fe}(\mathrm{II})$ and $\mathrm{Fe}$ (III) were localized in discrete biofilm microenvironments (Fig. 2b). Fe(III) was mainly associated with cell surfaces, while small amounts of $\mathrm{Fe}(\mathrm{II})$ was found in the extracellular space. Fe(III) was expected based on previously described $\mathrm{pH} / \mathrm{Eh}$ conditions (via confocal), however these conditions would likely shift prevent Fe(III) reduction to the ferrous form. The presence of ferrous iron implies that chemical microenvironments exist below the resolution limits of previously used chemically-sensitive probes, and that biofilm heterogeneity may be more complex than previously believed.

This study examines both the organization of microenvironments and their correlation to natural geochemical processes, but can also be extended to understand $P$. aeruginosa biofilms in a clinical context. Since this bacterium is also a known human pathogen, particularly with respect to chronic infection of the cystic fibrosis lung, applying these technologies to clinically-relevant biofilms may also highlight important details of iron acquisition/utilization processes during infection. To this end, the continued development of STXM and novel sample preparation methods appears promising. 
References

[1] R.C. Hunter and T.J. Beveridge, Appl Environ Microbiol 71 (2005) 2501

[2] R.C. Hunter and T.J. Beveridge, J Bacteriol. 187 (2005) 7619

[3] R.C. Hunter et al., Environ Sci Technol 42 (2008) 8766

[4] This research was supported by NSERC-Discovery, NSERC-PGS, and US-DOE. STXM was performed by Adam Hitchcock's team (McMaster University) and was supported by NSF, DMR, US-DOE, Dow Chemical, NSERC, and AFMnet.
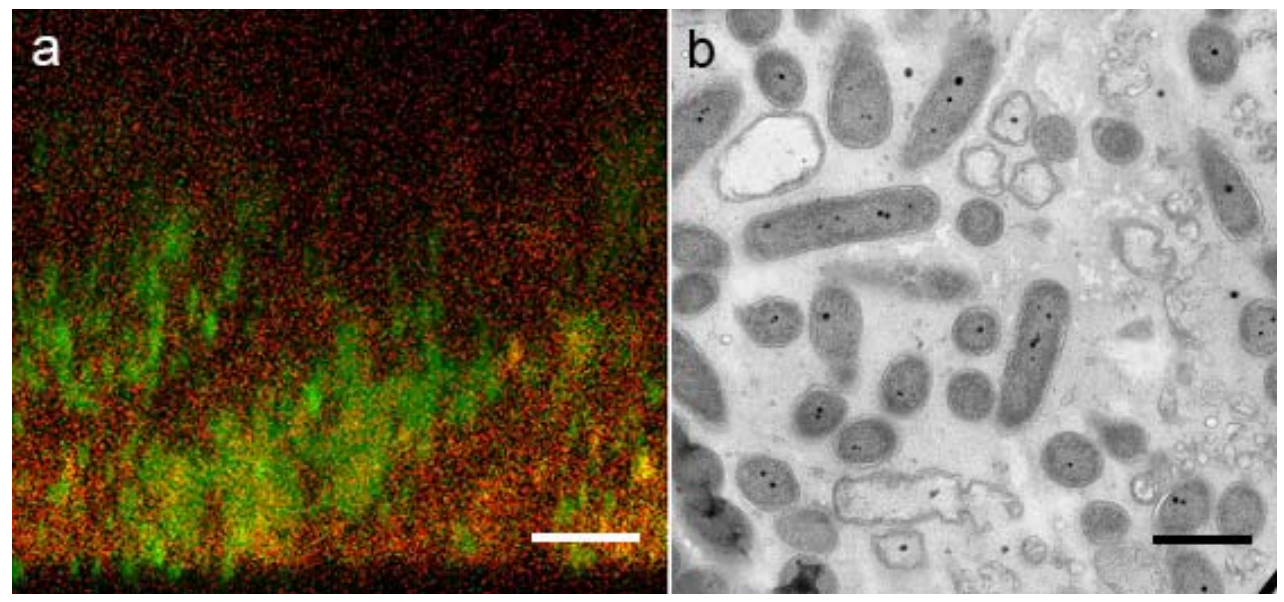

FIG. 1. a) Confocal image of a C-SNARF-treated biofilm (z-axis), showing $\mathrm{pH}$ heterogeneity. The ratio of green/red emission intensity is dependent on local $\mathrm{pH}$. Bar $=$ $10 \mu \mathrm{m}$. b) Freeze-substituted biofilm of $P$. aeruginosa showing a heterogeneous distribution of viable cells and extracellular polymers. Bar $=1 \mu \mathrm{m}$. These conditions are thought to drive complex metal-microbe interactions throughout biofilms.

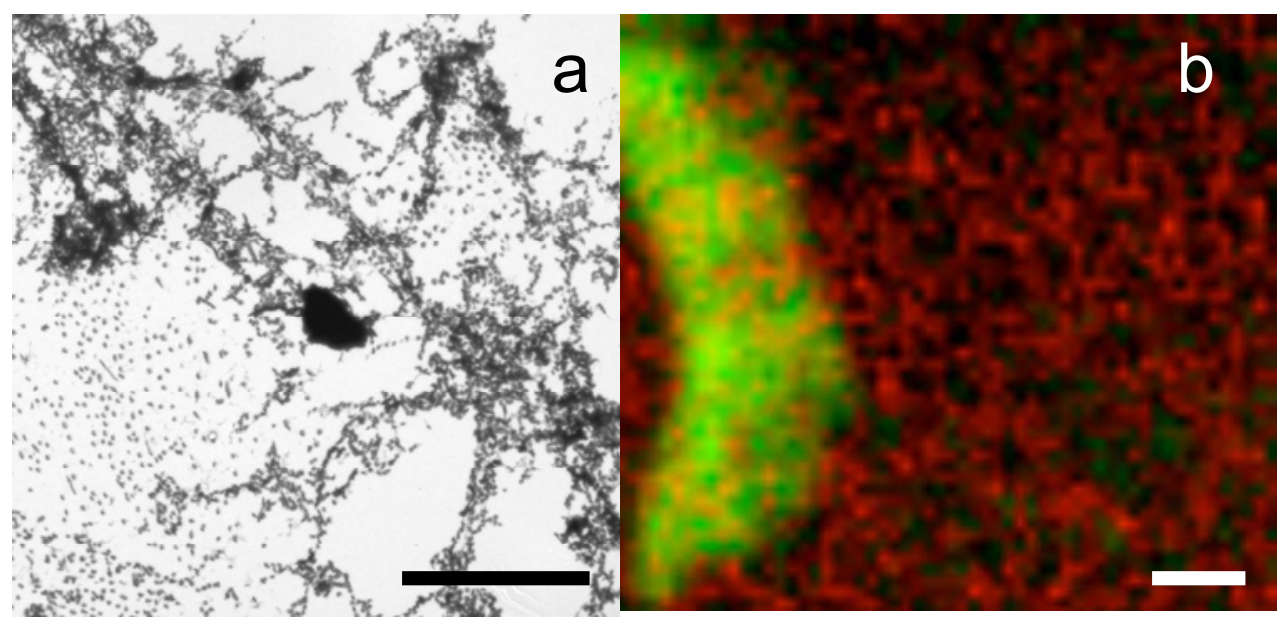

FIG. 2. a) TEM image of a freeze-dried cryo-section of a $P$. aeruginosa biofilm treated with $1 \mathrm{mM} \mathrm{Fe}\left(\mathrm{NO}_{3}\right)_{3}$. These samples were subsequently analyzed by STXM. Bar $=40$ $\mu \mathrm{m}$. b) Composite map of Fe(II) (red) and Fe(III) (green) derived from Fe 2p STXM imaging. Fe(III) is associated with the bacterial cell, while Fe(II) is partitioned into the extracellular milieu. Bar $=500 \mathrm{~nm}$. 